\title{
Control volume-radial basis function method for two-dimensional non-linear heat conduction and convection problems
}

\author{
C. A. Bustamante ${ }^{1}$, W. F. Flórez ${ }^{1}$, H. Power $^{2}$, M. Giraldo ${ }^{1}$ \\ \& A. F. Hill ${ }^{1}$ \\ ${ }^{1}$ Institute of Energy, Materials and Environment, \\ Universidad Pontificia Bolivariana, Colombia \\ ${ }^{2}$ School of Mechanical, Materials and Manufacturing Engineering, \\ University of Nottingham, $U K$
}

\begin{abstract}
An improvement to the traditional Finite Volume Method (FVM) for the solution of boundary value problems is presented. The new method applies the local Hermitian interpolation with Radial Basis Functions (RBF) as an interpolation scheme to the FVM discretization. This approach, called the Control Volume-Radial Basis Function (CV-RBF) method, uses an interpolation scheme based on the meshless Symmetric method, in which the numerical solution is approximated by employing the governing equation and the boundary condition operators. The RBF implemented is the Multiquadric (MQ) with a shape parameter found experimentally. The two-dimensional solutions to the Dirichlet problem for linear heat conduction, heat transfer in the Poiseuille flow and the non-linear conduction situations are obtained by the CV-RBF method. The numerical results are in agreement with the corresponding analytical and numerical solutions found in the literature.

Keywords: radial basis function, finite volume method, heat transfer, local Hermitian interpolation.
\end{abstract}

\section{Introduction}

The Finite Volume Method (FVM) has become one of the preferred discretization strategies in the field of transport phenomena. The interpolation scheme used to 
approximate the diffusive and convective fluxes on the volume faces is an important aspect for improving the FVM. The compilation of interpolation schemes done by Patankar [1] and later by Versteeg and Malalasekera [2] and Wesseling [3], has been enlarged to a variety of strategies with the aim of decreasing the truncation error preserving convergence, stability and consistency. In addition, interpolation schemes that are able to treat unstructured meshes are necessary to get solutions for the transport phenomena equations in domains with complex geometry.

To achieve an efficient and accurate approximation of the fluxes at the volume faces on unstructured meshes, several authors proposed schemes that use traditional FVM in conjunction with high order approximation methods, for instance, a two-dimensional polynomic approximation [4], the Least Square Reconstruction Technique (LSRT) [5] and the Gauss-Green Reconstruction technique (GGRT) $[6,7]$. In addition, the geometric treatment required to compute approximated fluxes is the cause of the high dependence of the FVM on the mesh configuration. A meshless interpolation scheme can be a good option to weaken this relationship. According to the above, the Radial Basis Function (RBF) collocation method is used here as an interpolation scheme, which results in a high order approximation [8] that is able to fit quasi-scattered data.

Currently the RBF collocation is widely used in the scattered data interpolation. In addition, it is the base of several meshless methods used to solve Partial Differential Equations (PDEs). The first attempt to develop a meshless method by RBF collocation was made by Kansa [9]. Kansa used the Multiquadric (MQ) function to obtain an accurate meshless solution to the advection-diffusion and Poisson equations without employing any special treatment for the advection term (upwinding), due to the high order of the resultant scheme and the intrinsic relationship between governing equations and the interpolation. This strategy involved all the nodes in the domain, therefore it produced a global full matrix. Although the Kansa's method was used by many authors to solve the Laplace, the Poisson, the Helmotz and Parabolic equations, showing better accuracy compared to traditional methods [10-13], Kansa and Hon [14] concluded that the matrix ill-conditioning worsens as the number of nodes increases. In the case of using the MQ function, a singular matrix is obtained for some values of the shape parameter. Fasshauer [15] used the Hermite interpolation to construct an approximated function that resulted in a symmetric matrix and therefore a lesser amount of computation. He concluded that, for the PDEs solved, the Hermite interpolation or the Symmetric method perform slightly better than the Kansa or Unsymmetric methods. Jumarhon et al. [16] obtained a similar improvement using the Symmetric method and Power and Barraco [17] obtained better results by employing the Symmetric method for a variety of problems, including the convection diffusion equation.

Due to the ill-conditioned matrix of the global RBF collocation, it was implemented locally to improve the traditional methods for solving PDEs. The Finite Difference Method (FDM) was modified by Wright and Fornberg [8] to obtain higher order approximation by employing RBF Hermite interpolation. In the Finite 
Volume Method (FVM), the RBF collocation was first implemented as an interpolation scheme by Moroney and Turner [18] to solve the two-dimensional nonlinear anisotropic diffusion equation and later, by Moroney and Turner [19] for the three-dimensional case in the same situation. Recently Orsini et al. [20] solved the diffusion convection equation by the FVM in conjunction with the RBF Hermite interpolation scheme. They had the convective and diffusive fluxes in terms of neighboring node values according to the Symmetric method and called this strategy the Control Volume-Radial Basis Function (CV-RBF).

In this work, the CV-RBF method is used to solve the two-dimensional heat equation for steady situations. In the next section the mathematical model that governs the steady heat convection and conduction is presented. After, the CV-RBF method is explained and the final discrete equation for the generic convectiondiffusion problem is obtained. Finally, the numerical result are presented and compared with numerical and analytical solutions found in literature.

\section{Governing equations}

From the energy balance in an incompressible continuum medium, the heat equation (1) is obtained by subtracting the mechanical energy, expressing the internal energy in terms of the temperature and neglecting the accumulation and viscous dissipation terms. In this expression $\rho$ is the medium density, $\hat{C}_{p}$ is the specific heat at constant pressure, $\vec{v}$ is the velocity field and $S$ is the source term, which can depend on temperature and space.

$$
\rho \hat{C}_{p} v_{j} \frac{\partial T}{\partial x_{j}}=-\frac{\partial q_{j}}{\partial x_{j}}+S(T, \vec{x})
$$

The molecular phenomena responsible of the conduction heat flux $\vec{q}$ in an isotropic system can be quantified on a macroscopic level by Fourier's Law (2), where $k$ is the thermal conductivity.

$$
q_{i}=-k(T) \frac{\partial T}{\partial x_{i}}
$$

\section{Control volume-radial basis function}

The CV-RBF method improves the traditional FVM by an interpolation scheme based on the Hermitian RBF. In this section, the formulation of the CV-RBF method developed by Orsini et al. [20] applied to the heat equation is presented.

\subsection{Finite Volume Method discretization}

The discretization process is applied to the heat equation (1) after substituting the Fourier's law (2) in it. First, the governing equation is integrated over a generic volume and the divergence theorem is applied to obtained the expression (3), where 
$\Omega$ and $\partial \Omega$ are the finite volume and its surrounding surface, respectively.

$$
\oint_{\partial \Omega} k \frac{\partial T}{\partial x_{i}} n_{i} d \partial \Omega-\oint_{\partial \Omega} \rho \hat{C}_{p} v_{i} T n_{i} d \partial \Omega=-\int_{\Omega} S d \Omega
$$

Using non-overlapping polygonal volumes, the surface integral can be expressed as the sum of the integrals over each of the $m$ volume faces. Then, the surface and volume integrals have to be approximated by a numerical method. In this case for simplicity and according to traditional FVM, the mid-point rule is employed both for the surface and volume integrals. The resultant expression (4) is the final FVM discretized equation. The volume and surface geometric centers are $\vec{x}_{p}$ and $\vec{x}_{l}$, respectively.

$$
\left.\sum_{l=1}^{m}\left(k \frac{\partial T}{\partial x_{i}} n_{i} \Delta \partial \Omega\right)\right|_{\vec{x}=\overrightarrow{x_{l}}}-\left.\sum_{l=1}^{m}\left(\rho \hat{C}_{p} v_{i} T n_{i} \Delta \partial \Omega\right)\right|_{\vec{x}=\overrightarrow{x_{l}}}=-S\left(\vec{x}_{p}\right) \Delta \Omega
$$

To complete the solution procedure, the dependent variable and its gradient, at the surface central points, have to be approximated in terms of the neighbor volume central nodes according to an interpolation scheme.

\subsection{Local Hermitian Interpolation scheme}

The Hermitian Interpolation scheme is a symmetrical RBF collocation technique used to solve PDEs. The RBF main feature is the only dependence in Euclidian distance between the evaluation point $\vec{x}$ and the trial nodes $\vec{\xi}$. Another characteristic is the monotonically increasing or decreasing of the function with the distance from the evaluation point. Here, the RBF used is the Multiquadric MQ function (5), where $r=\|\vec{x}-\vec{\xi}\|$ is the Euclidian distance and $c$ is the shape parameter which allows to modified the slope of the function. The MQ function converges exponentially and is conditionally positive defined of order $m>0$, since a $m-1$ order polynomial has to be added to get a non-singular interpolation matrix. In this case, $m=1$ and the shape parameter is obtained by numerical experiments.

$$
\Psi(r)=\left(r^{2}+c^{2}\right)^{m / 2}
$$

A boundary value problem is considered for the dependent variable $\phi$. The problem is defined by equations (6) and (7), where $L$ and $B$ are linear differential operators that apply over the domain $\Omega$ and the boundary $\partial \Omega$, respectively.

$$
\begin{aligned}
& L[\phi(\vec{x})]=f(\vec{x}) \\
& B[\phi(\vec{x})]=g(\vec{x})
\end{aligned}
$$

In RBF collocation strategies to solve PDEs are two basic procedures: Kansa's Method [9] and Symmetric Method [21]. The Hermite Interpolation scheme is used on the Symmetric method. A variation of the latter Symmetric method was developed by Orsini et al. [20], in which the Hermite Interpolation applies locally. 


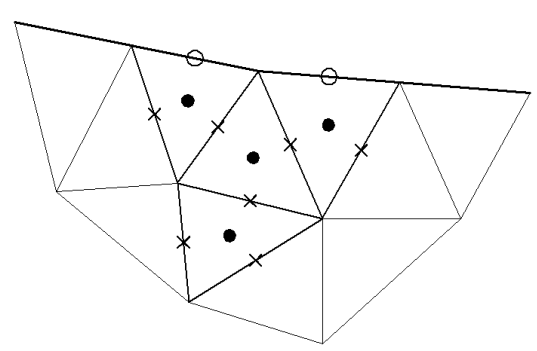

Figure 1: Node locations inside the stencil: • central nodes (NP), ○ boundary points $(\mathrm{NB}), \times$ internal nodes $(\mathrm{NPDE})$.

In this case, it is necessary to define a subdomain which contains the nodes. In the CV-RBF method, the subdomain is called stencil and it contains the central volume and at least one neighbor volume. The stencil includes $N P$ central nodes or points where the variable is unknown, $N B$ boundary points where the boundary operator is applied and NPDE internal locations where the governing PDE operator is enforced. The stencil used here was called one stencil one face by Orsini et al. [20] and is shown in Figure 1 in the case of a two-dimensional triangular unstructured mesh.

Unlike the global Symmetric method, the approximated function is given by the equation (8), where $L_{\xi}$ and $B_{\xi}$ are the differential operators acting on $\Psi$ view as a function of $\vec{\xi}, r$ is the number of polynomial terms and $N T$ is the total number of points inside the stencil. By replacing the approximation function in the boundary condition (7) and the governing PDE (6) and evaluating the resultant formulae at the respective points, a linear system $A \alpha=B$ is obtained. The symmetric interpolation matrix $A$ is given by the equation (9) and the column vector $B$ by the expression (10).

$$
\begin{aligned}
\phi(\vec{x})= & \sum_{j=1}^{N P} \alpha_{j} \Psi\left(\left\|\vec{x}-\vec{\xi}_{j}\right\|\right)+\sum_{j=N P+1}^{N P+N B} \alpha_{j} B_{\xi}\left[\Psi\left(\left\|\vec{x}-\vec{\xi}_{j}\right\|\right)\right] \\
& +\sum_{j=N P+N B+1}^{N P+N B+N P D E} \alpha_{j} L_{\xi}\left[\Psi\left(\left\|\vec{x}-\vec{\xi}_{j}\right\|\right)\right]+\sum_{j=1}^{r} \alpha_{j+N T} P_{m-1}^{j}(\vec{x})
\end{aligned}
$$

$$
A=\left(\begin{array}{cccc}
\boldsymbol{\Psi} & B_{\xi}[\mathbf{\Psi}] & L_{\xi}[\mathbf{\Psi}] & P_{m-1} \\
B_{x}[\boldsymbol{\Psi}] & B_{x} B_{\xi}[\mathbf{\Psi}] & B_{x} L_{\xi}[\mathbf{\Psi}] & B_{x}\left[P_{m-1}\right] \\
L_{x}[\boldsymbol{\Psi}] & L_{x} B_{\xi}[\mathbf{\Psi}] & L_{x} L_{\xi}[\boldsymbol{\Psi}] & L_{x}\left[P_{m-1}\right] \\
P_{m-1}^{T} & B_{x}\left[P_{m-1}^{T}\right] & L_{x}\left[P_{m-1}^{T}\right] & 0
\end{array}\right)
$$




$$
B=\left(\begin{array}{c}
\phi_{v o l} \\
g(\vec{x}) \\
f(\vec{x}) \\
0
\end{array}\right)
$$

The above linear equation system can not be solved because the column vector $B$ includes, in $\phi_{v o l}$, the $N P$ unknown values of the variable at the central nodes contained in the stencil. However, the coefficient $\alpha$ in terms of the unknown variable values can be used to interpolate the variable and its gradient at the locations required by the FVM discretization. To do this, the approximated gradient is obtained by deriving the function (8). Then, the approximated variable and its gradient can be expressed in matrix notation by equations (11) and (12). The matrices $C_{1}$ and $C_{2}$ are known from the geometrical information, since they include the RBFs and the resultant functions after applying operators to them.

$$
\begin{aligned}
\phi(\vec{x}) & =\left[C_{1 l}\right]^{T}[\alpha] \\
\frac{\partial \phi(\vec{x})}{\partial x_{i}} & =\left[C_{2 i l}\right]^{T}[\alpha]
\end{aligned}
$$

To solve the heat equation (1) by the CV-RBF method, it is necessary to replace the variable (11), in this case temperature, and its gradient (12) obtained by the local Hermitian Interpolation in the FVM discretized equation (4). The expression obtained from this procedure is the final CV-RBF discretized equation (13). By applying the expression (13) to each one of the finite volumes, a linear or nonlinear system of equation is obtained.

$$
\begin{array}{r}
\left(\left.\sum_{l=1}^{m}\left(k C_{2 i}^{T} n_{i} \Delta \partial \Omega\right)\right|_{\vec{x}=\vec{x}_{l}}-\left.\sum_{l=1}^{m}\left(\rho \hat{C}_{p} C_{1}^{T} v_{i} n_{i} \Delta \partial \Omega\right)\right|_{\vec{x}=\vec{x}_{l}}\right) A^{-1} B \\
-S\left(\vec{x}_{p}\right) \Delta \Omega=0
\end{array}
$$

In the non-linear case, the Newton-Rapshon method is used to solve the resultant equation system by computing numerically the Jacobian matrix by the central difference scheme. To applied the Hermitian Interpolation, a linear operator $L$ has to be constructed from the governing equation. In the case of non-linear heat conduction, the Laplace operator is obtained according to the expression (14). The superindex $n$ refers to the quantity values at the present iteration.

$$
L_{x}\left(T^{n}\right)=\frac{\partial^{2} T^{n}}{\partial x_{i} \partial x_{i}}=\frac{1}{k\left(T^{n-1}\right)}\left(-\frac{\partial k\left(T^{n-1}\right)}{\partial T} \frac{\partial T^{n-1}}{\partial x_{i}} \frac{\partial T^{n-1}}{\partial x_{i}}-S(\vec{x})\right)
$$

\section{Numerical results}

The CV-RBF method is applied to solve the heat equation (1) on two dimensional domains for the linear and non-linear heat conduction in a solid, and to the thermal 


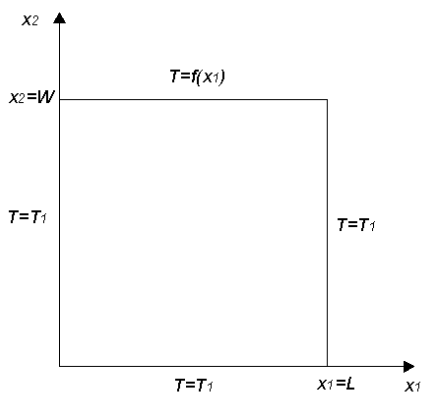

(a)

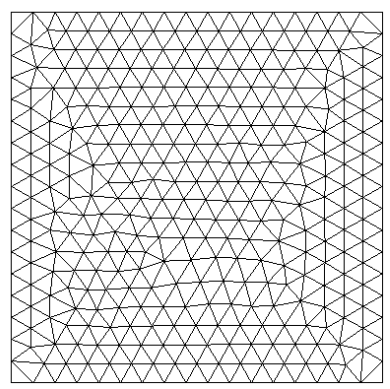

(b)

Figure 2: Domain characteristics, (a) geometry, (b) unstructured mesh.

developing profile in the plane Poiseuille flow. The results are compared to the analytical solution for case of conduction, and to the numerical results found in literature for the convection problem.

\subsection{Dirichlet problem}

A rectangular domain of $W \times L$ which represents a solid thin plate is considered. In Figure 2 (a), the geometry and the boundary conditions are shown. The governing equation is reduced to the expression (15) in terms of the variable $\theta=T-T_{1}$ where $T_{1}$ is the temperature in all boundaries except at $x_{2}=W$.

$$
\frac{\partial}{\partial x_{j}}\left(k \frac{\partial \theta}{\partial x_{j}}\right)=-S\left(x_{1}, x_{2}\right)
$$

The boundary condition at $x_{2}=W$ is given by the expression (16) and the respective analytical solution is the equation (17), where $\theta_{m}=T_{2}-T 1$ and $T_{2}$ is the maximum temperature value. The shape parameter for the MQ function is equal to the mean distance between central and neighbor nodes.

$$
\begin{gathered}
f\left(x_{1}\right)=T_{2} \sin \left(\frac{n \pi x_{1}}{L}\right) \\
\theta\left(x_{1}, x_{2}\right)=\theta_{m} \sin \left(\frac{\pi x_{1}}{L}\right) \frac{\sinh \left(\pi x_{2} / L\right)}{\sinh (\pi W / L)}
\end{gathered}
$$

The unstructured mesh used on the domain, in the case of 384 volumes, is shown in Figure 2 (b). First, the numerical solutions, the global Root Mean Square (RMS) errors and the global maximum errors are obtained for different mesh densities. The results are presented in Table 1 where the convergence of the method can be seen for the linear conduction problem.

The numerical results obtained for a mesh of 384 volumes are shown in Figure 3 , on three domain lines with $x_{1}$ constant. The relative error increases near 
Table 1: RMS and maximum error in the solution of linear heat conduction problem.

\begin{tabular}{|c|c|c|}
\hline Mesh size & $\epsilon_{\max }$ & $\epsilon_{R M S}$ \\
\hline 32 vol & 0.0422 & 0.0165 \\
\hline 100 vol & 0.0245 & 0.0105 \\
\hline 384 vol & 0.0136 & 0.0042 \\
\hline 882 vol & 0.0053 & 0.0013 \\
\hline
\end{tabular}

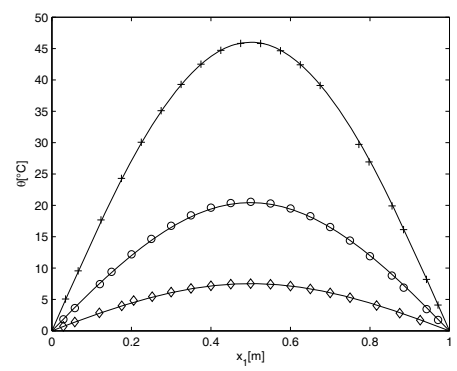

(a)

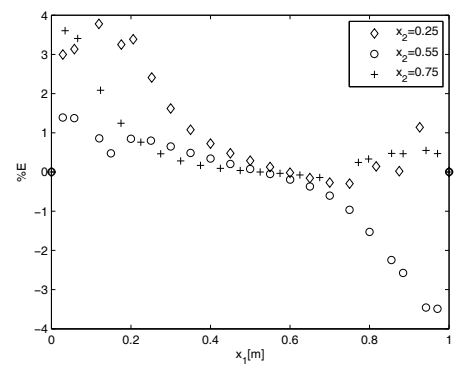

(b)

Figure 3: Solution of the linear heat conduction problem, (a) temperature profile on lines $x_{2}=0.25(\diamond), x_{2}=0.50(o)$ and $x_{2}=0.75(+),(b)$ relative error.

boundaries but its magnitude is not greater than $4 \%$. According to these results, the CV-RBF method can solve accurately the linear conduction problem with Dirichlet boundary conditions.

\subsection{Non-linear conduction}

A hypothetical heat conduction situation is considered in a solid material with a thermal conductivity in terms of temperature. The phenomena is modelled by the equation (15) where $\theta=T-T_{1}, k=T^{1.3}$ and $S$ is obtained by replacing the imposed solution (18) on the governing PDE. The problem geometry and the boundary conditions are the same as those presented in the Figure 2, with $f\left(x_{1}\right)=$ $T_{1}$.

$$
T\left(x_{1}, x_{2}\right)-T_{\infty}=100 T_{\infty} x_{1} x_{2}\left(1-x_{1}\right)\left(1-x_{2}\right) e^{-\left(x_{1}^{2}+x_{2}^{2}\right)}
$$

The numerical solution is obtained using the MQ function with $m=1$ and a shape parameter $c=0.1 h$, where $h$ is the mean distance between central nodes in the stencil. A $30 \times 30$ structured mesh is used in the solution. The Figure 4 shows the exact solution contour (left) and the numerical solution contour (right). 


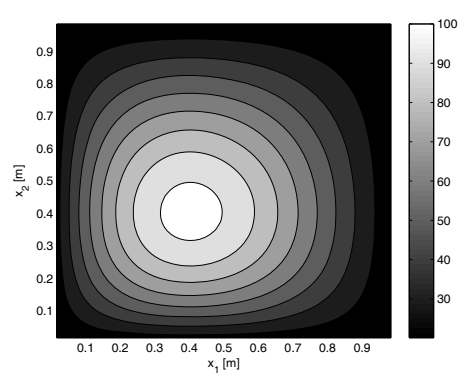

(a)

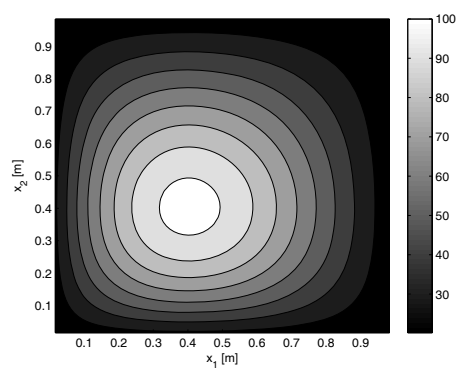

(b)

Figure 4: Temperature contours for the non-linear heat conduction problem: (a) analytical solution; (b) numerical solution.

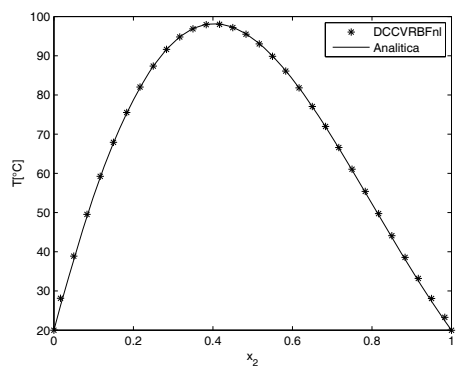

(a)

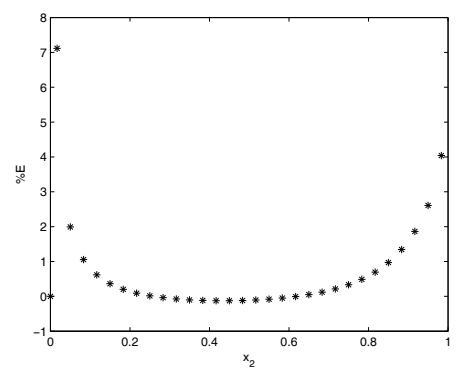

(b)

Figure 5: Comparison between analytical and numerical solution of the non-linear heat conduction problem at the line $x_{1}=0.5$ : (a) temperature profile; (b) relative error.

Also, the temperature profile and the relative error at the line $x_{1}=0.5$ are shown in Figures 5 (a) and (b), respectively. The figures mentioned show the good agreement between the analytical and numerical solution. The relative error, as in the linear case, increases near boundaries without exceeding $8 \%$. This error is presented locally and does not affect the global RMS error which is $0.54 \%$.

\subsection{Temperature profile in the plane Poiseuille flow}

An incompressible Newtonian fluid flows in the $x_{1}$ direction between two plates as shown in Figure 6. A hydraulic developed flow in steady state and a field velocity independent of temperature are assumed. According to this, the known velocity field is the plane Poiseuille flow described by equation (19). The fluid selected is air with constant properties and the maximum velocity is set to get a global Peclet number close to 1200 . 


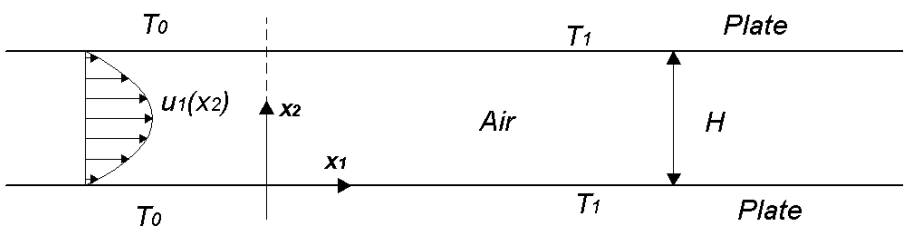

Figure 6: Geometrical configuration for the heat transfer in plane Poiseuille flow.

$$
u_{1}\left(x_{2}\right)=\left(u_{1}\right)_{\max }\left(1-\left(\frac{x_{2}-0.5 H}{0.5 H}\right)^{2}\right)
$$

The governing equation is the expression (1) without including the source term. The boundary conditions are given by the expressions from (20) to (22) and the domain is divided by a $30 \times 90$ structured mesh. The MQ with $m=1$ is used and the shape parameter is computed as $c=0.1 h$. The analytical solution is known as the Graetz problem in rectangular coordinates, but there is not a close form of the solution. Therefore, the results are compared to those obtained by Divo and Kassab [22] employing an RBF collocation method.

$$
\begin{gathered}
T\left(0, x_{2}\right)=T_{0} \\
T\left(x_{1}, 0\right)=T\left(x_{1}, H\right)=T_{1} \\
\frac{\partial T\left(L, x_{2}\right)}{\partial x_{1}}=0
\end{gathered}
$$

The thermal profile obtained is shown in Figure 7 (a). The temperature distribution agrees with the physical phenomena involved here, since near the plates the temperature is $T_{1}$ and it increases to $T_{0}$ close to the center line. In the Figure 7 (b) are presented the temperature profiles on several $x_{1}$ constant lines and can

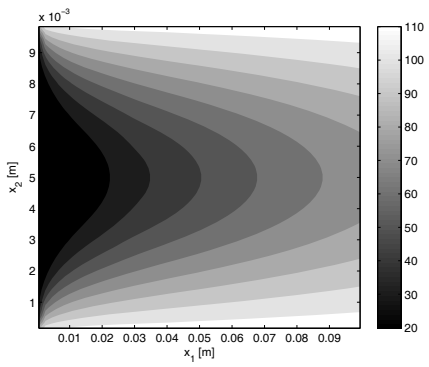

(a)

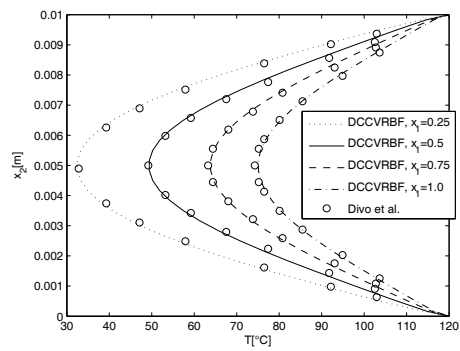

(b)

Figure 7: Temperature profile in the plane Poiseuille flow: (a) Temperature contour; (b) Temperature profiles at $x_{1}$ constant lines. 
be seen the agreement between the numerical results obtained and those found in literature.

\section{Conclusions}

The heat equation was solved accurately for basic two-dimensional situations by the CV-RBF method. This is a successful application of the method considering the equations solved and the coupling to the Newton-Rapshon method.

The CV-RBF method is an improvement to the traditional FVM due to the geometric treatment versatility. Both the results in unstructured and structured meshes are in agreement to the reference data.

The MQ function with $m=1$ was used to solve accurately the heat equation. Although, the solution depends on the shape parameter and there is not all-purpose values or expressions for it. Hence, obtaining the shape parameter is an aspect to be improved in the method.

The main strengths of the CV-RBF method are the capability to deal with unstructured meshes, the high order of the interpolation scheme and the intrinsic upwind differencing.

\section{References}

[1] Patankar, S., Numerical heat transfer and fluid flow. Computational and Physical Processes in Mechanics and Thermal Sciences, Taylor \& Francis, 1980.

[2] Versteeg, H.K. \& Malalasekera, W., Introduction to computational fluid dynamics: the finite volume method. Longman Scientific \& Technical, 1995.

[3] Wesseling, P., Principles of computational fluid dynamics. Computational Mathematics, Springer, 2001.

[4] Abgrall, R., On essentially non-oscillatory schemes on unstructured meshes. Journal of Computational Physics, 114(1), pp. 45-98, 1994.

[5] Jayantha, P.A. \& Turner, I.W., A second order control-volume-finite-element least-squares strategy for simulating diffusion in strongly anisotropic media. Journal of Computational Physics, 23(1), pp. 1-16, 2005.

[6] Truscott, S.L. \& Turner, I.W., An investigation of the accuracy of the control volume-element based on triangular prismatic elements for simulating diffusion in anisotropic media, part b: Fundamentals. Numerical Heat Transfer, 46(1), pp. 243-268, 2004.

[7] Manzini, G. \& Putti, M., Mesh locking effects in the finite volume solution of 2-d anisotropic difusin equations. Journal of Computacional Physics, 220(1), pp. 751-771, 2007.

[8] Wright, G. \& Fornberg, B., Scattered node compact finite difference-type formulas generated from radial basis functions. Journal of Computational Physics, 212(1), 2006. 
[9] Kansa, E.J., Multiquadrics -a scattered data approximation scheme with applications to computational fluid dynamics-ii solution to parabolic, hyperbolic and elliptic partial differential equations. Computers \& Mathematics with applications, 19(8-9), pp. 127-145, 1990.

[10] Zerroukat, M., Power, H. \& Chen, C.S., A numerical method for heat transfer problems using collocation and radial basis functions. International Journal for Numerical Methods in Engineering, 42(7), pp. 1263-1278, 1998.

[11] Li, J., Cheng, A.H.D. \& Chen, C.S., A comparison of efficiency and error convergence of multiquadric collocation method and finite element method. Engineering Analysis with Boundary Elements, 27(3), pp. 251-257, 2003.

[12] Cheng, A.H.D. \& Cabral, J.J.S.P., Direct solution of ill-posed boundary value problems by radial basis function collocation method. International Journal for Numerical Methods in Engineering, 64(1), pp. 45-64, 2005.

[13] Chantasiriwan, S., Cartesian grid methods using radial basis functions for solving poisson, helmholtz, and diffusionconvection equations. Engineering Analysis with Boundary Elements, 28(12), pp. 1417-1425, 2004.

[14] Kansa, E.J. \& Hon, Y.C., Circumventing the ill- conditioning problem with multiquadric radial basis functions: Applications to elliptic partial differential equations. Computers \& Mathematics with applications, 39(1), pp. 123-137, 2000.

[15] Fasshauer, G., Solving partial differential equations by collocation with radial basis functions. Proceedings of Chamonix, Vanderbilt University Press, pp. 1-8, 1997.

[16] Jumarhon, B., Amini, S. \& Chen, K., The hermite collocation method using radial basis functions. Engineering Analysis with Boundary Elements, 24(78), pp. 607-611, 2000.

[17] Power, H. \& Barraco, V., A comparison analysis between unsymmetric and symmetric radial basis function collocation methods for the numerical solution of partial differential equations. Computers \& Mathematics with Applications, 43(3-5), pp. 551-583, 2002.

[18] Moroney, T.J. \& Turner, I.W., A finite volume method based on radial basis functions for two-dimensional nonlinear diffusion equations. Applied Mathematical Modelling, 30(10), 2006.

[19] Moroney, T.J. \& Turner, I.W., A three dimensional finite volume method based on radial basis functions for the accurate computacional modelling of nonlinear diffusion equations. Journal of Computational Physics, 225(2), 2007.

[20] Orsini, P., Power, H. \& Morovan, H., Improving volume element methods by meshless radial basis function techniques. Computer modelling in Engineering and Sciences, 769(1), pp. 1-21, 2008.

[21] Fedoseyev, A.I., Friedman, M. \& Kansa, E.J., Improve multiquadric method for elliptic partial differential equations via pde collocation on the boundary. Computers \& Mathematics with applications, 43(3-5), pp. 439-455, 2002.

[22] Divo, E. \& Kassab, A.J., A meshless method for conjugate heat problems. Engineering Analysis with Boundary Elements, 29(1), pp. 136-149, 2005. 